\title{
Are the strengths of solar cycles determined by converging flows towards the activity belts?
}

\author{
R. H. Cameron and M. Schüssler
}

\begin{abstract}
Max-Planck-Institut für Sonnensystemforschung, Max-Planck-Str. 2, 37191 Katlenburg-Lindau, Germany
e-mail: [cameron; schuessler] @mps.mpg.de
\end{abstract}

Received 28 June 2012 / Accepted 19 October 2012

\section{ABSTRACT}

\begin{abstract}
It is proposed that the observed near-surface inflows towards the active regions and sunspot zones provide a nonlinear feedback mechanism that limits the amplitude of a Babcock-Leighton-type solar dynamo and determines the variation of the cycle strength. This hypothesis is tested with surface flux transport simulations including converging latitudinal flows that depend on the surface distribution of magnetic flux. The inflows modulate the build-up of polar fields (represented by the axial dipole) by reducing the tilt angles of bipolar magnetic regions and by affecting the cross-equator transport of leading-polarity magnetic flux. With flux input derived from the observed record of sunspot groups, the simulations cover the period between 1874 and 1980 (corresponding to solar cycles 11 to 20). The inclusion of the inflows leads to a strong correlation of the simulated axial dipole strength during activity minimum with the observed amplitude of the subsequent cycle. This in agreement with empirical correlations and in line with what is expected from a Babcock-Leighton-type dynamo. The results provide evidence that the latitudinal inflows are a key ingredient in determining the amplitude of solar cycles.
\end{abstract}

Key words. Sun: dynamo - Sun: activity - Sun: surface magnetism

\section{Introduction}

The correlation between the amplitude of the solar polar field around activity minimum and the strength of the subsequent cycle (e.g., Schatten et al. 1978; Choudhuri 2008; Cameron et al. 2010; see, however, Layden et al. 1991) suggests that the Sun's axial dipole field as observed around activity minimum reflects the source for the generation of the toroidal magnetic flux of the subsequent cycle, as opposed to being a mere epiphenomenon of an otherwise fully hidden subsurface dynamo process (Cameron \& Schüssler 2007; Schüssler 2007). Such a role of the polar field is a key feature of the Babcock-Leighton (BL) dynamo model (see Charbonneau 2010, for a review of solar dynamo theory). In this type of dynamo, the poloidal magnetic field results from the systematic tilt (with respect to the azimuthal/longitudinal direction) of sunspot groups and bipolar magnetic regions in combination with the subsequent redistribution of their magnetic fluxes by near-surface flows. The crucial mechanism for the reversal of the Sun's global dipole field is the preferred transport of preceding-polarity flux of bipolar magnetic regions across the equator into the other solar hemisphere (Cameron \& Schüssler 2007), which leads to the reversal of the polar fields and the build-up of an axial dipole field of opposite polarity. Toroidal flux is then generated by the action of differential rotation winding up poloidal field lines threading the Sun.

So far we have described the BL dynamo as a linear process. In addition, at least one nonlinear feedback mechanism is required that limits the amplitude of the generated magnetic field and controls the variation of the cycle amplitude. In the framework of the BL dynamo, potential feedback mechanisms include the back-reaction of the magnetic field on 1) differential rotation; 2 ) the tilt angles of sunspot groups; and 3) cross-equator transport of magnetic flux.
Concerning possibility 1), the only empirically wellestablished cycle-related variation of the differential rotation are zonal flows of a few $\mathrm{ms}^{-1}$, which at low latitudes are associated with the activity belts (Howard \& Labonte 1980; Zhao \& Kosovichev 2004; Gizon 2004; Gizon \& Birch 2005). Clear evidence for a more global reaction on differential rotation that would affect the generation of the toroidal magnetic field has not been obtained so far.

As to mechanism 2), the back-reaction on the tilt angles of sunspot groups, Dasi-Espuig et al. (2010) found cycle-to-cycle variations of the average tilt angle, which are anti-correlated with the cycle strength: strong cycles show smaller average tilt angles than weak cycles. Since the tilt angles affect the strength of the source for the poloidal field in the BL dynamo, their anticorrelation with cycle strength provides a possible nonlinear saturation mechanism. Cameron et al. (2010) included the observed tilt-angle variations in a surface flux transport (SFT) simulation with sources taken from the combined Royal Greenwich Observatory (RGO) and the Solar Optical Observing Network (SOON) sunspot records. Considering the period for which tiltangle data are available (1913-1986), they found that the maximum of the polar field (occurring around activity minimum of a given cycle) in the SFT simulation is correlated with the observed strength of the subsequent activity cycle. This suggests that the anti-correlation of the average tilt angle with cycle strength contributes to the variations of the cycle amplitudes and thus to the nonlinear feedback by which the solar dynamo saturates at the observed levels.

Which physical process could lead to the observed anticorrelation? One possibility is a change in the strength or effectiveness of the Coriolis force that tilts magnetic flux tubes rising in the convection zone (e.g., D'Silva \& Choudhuri 1993; Fan et al. 1994; Caligari et al. 1995, 1998; Fan 2009). In the 
absence of sufficient observational information about the subsurface structure and dynamics of the magnetic field, it is difficult to evaluate this mechanism. Another possible process is more amenable to observation and quantitative test: tilt-angle changes caused by the redistribution of magnetic flux after emergence by near-surface flows resulting from the presence of the magnetic field. A well-observed example of such flows are the inflows towards active regions and the activity belts (e.g., Haber et al. 2002; Zhao \& Kosovichev 2004; Gizon 2004; Gizon \& Rempel 2008; Švanda et al. 2008; González Hernández et al. 2010), possibly resulting from a temperature deficit due to the excess radiance of small-scale magnetic flux concentrations (Spruit 2003; Gizon \& Rempel 2008). Collectively, these inflows drive mean flows towards the activity belts and zonal flows, whose amplitudes depend on the amount of magnetic flux present in active regions, i.e., on the overall strength of a cycle. The transport of active-region flux by both the converging latitudinal flows and the longitudinal zonal flows tends to reduce the tilt angles of the corresponding bipolar magnetic regions. This mechanism was recently studied by Jiang et al. (2010) with SFT simulations including an ad-hoc model of the inflows in terms of latitudinal flows towards the activity belts. Considering individual bipolar regions as well as synthetic solar cycles, they found that the inflows reduce the lateral separation of the magnetic polarities, leading to a weakening of the contribution of a given bipolar region to the polar field. Building on these results, Jiang et al. $(2011 \mathrm{a}, \mathrm{b})$ included cycle-dependent tilt angles in their reconstruction of the evolution of the surface flux and open heliospheric flux since 1700 .

There is another aspect of the converging inflows towards active regions, which is related to possibility 3 ) for a nonlinear effect listed further above: a feedback on the cross-equator transport of magnetic flux. For active regions not too far from the equator, the inflows often extend over the equator (see, e.g., Fig. 18 in Gizon et al. 2010), which enhances the otherwise purely diffusive cross-equator transport of preceding-polarity flux and, therefore, tends to increase the resulting polar field. This effect is weaker for strong cycles because then active regions start to emerge at higher latitudes and the activity maximum is reached earlier (the Waldmeier effect), at a time when the activity belts are farther away from the equator. Therefore, this represents a negative feedback on the growth of the polar field.

In this paper, we present results of SFT simulations including mean latitudinal flows towards the activity belts, which were determined self-consistently from the simulated distribution of magnetic flux, thus introducing a nonlinear element into the SFT model. Using the RGO/SOON sunspot group data to determine the flux in emerging bipolar magnetic regions, we followed the evolution of the surface flux from 1874 until 1980. In order to evaluate the possible relevance of the nonlinearity for determining the cycle strength in the framework of a BL dynamo, we considered the correlation between the maximum of the magnetic flux associated with the axial dipole component in a given cycle resulting from the SFT simulation with the observed amplitude of the subsequent cycle.

The advantage of the approach chosen here is that it deals exclusively with observationally constrained near-surface processes and thus does not require a complete dynamo model in order to evaluate the potential of the nonlinearity. While the surface evolution addressed by SFT simulations is constrained by observations, a full dynamo model involves assumptions of the subsurface dynamics, which at present are essentially unconstrained.

\section{Surface flux transport simulation}

\subsection{Transport equation}

We used the SFT code of Baumann et al. (2004) in the version described by Jiang et al. (2010) to simulate the evolution of the magnetic flux at the solar surface under the influence of largescale surface flows (differential rotation and meridional flow) and a diffusivity representing the random motion of the magnetic flux elements due to the (numerically unresolved) supergranular flows. The magnetic field at the solar surface is assumed to be radial. The governing equation of the SFT model is

$$
\begin{aligned}
\frac{\partial B}{\partial t}= & -\omega(\lambda) \frac{\partial B}{\partial \phi}-\frac{1}{R_{\odot} \cos \lambda} \frac{\partial}{\partial \lambda}\{[v(\lambda)+\Delta v(\lambda, t)] B \cos \lambda\} \\
& +\eta_{\mathrm{H}}\left[\frac{1}{R_{\odot} \cos \lambda} \frac{\partial}{\partial \lambda}\left(\cos \lambda \frac{\partial B}{\partial \lambda}\right)+\frac{1}{R_{\odot}^{2} \cos ^{2} \lambda} \frac{\partial^{2} B}{\partial \phi^{2}}\right] \\
& +S(\lambda, \phi, t)+D\left(\eta_{r}\right)
\end{aligned}
$$

where $B(\lambda, \phi)$ denotes the radial component of the magnetic field, $\lambda$ is the heliographic latitude, and $\phi$ is the heliographic longitude. The expressions for the synodic differential rotation (from Snodgrass 1983),

$\omega(\lambda)=13.38-2.30 \sin ^{2} \lambda-1.62 \sin ^{4} \lambda[\%$ day $]$,

and for the time-independent part of the meridional flow (cf. van Ballegooijen et al. 1998),

$v(\lambda)= \begin{cases}11 \sin \left(\pi \lambda / 75^{\circ}\right) \mathrm{m} \mathrm{s}^{-1} & \text { where }|\lambda| \leq 75^{\circ} \\ 0 & \text { otherwise, }\end{cases}$

as well as the choice of the horizontal and radial diffusivities (Baumann et al. 2006), $\eta_{\mathrm{H}}=250 \mathrm{~km}^{2} \mathrm{~s}^{-1}$ and $D=0$, were the same as used in Cameron et al. (2010). The velocity $\Delta v(\lambda, t)$ in Eq. (1) represents the longitude-averaged inflows toward the activity belts, which are described by a magnetic-field-dependent local perturbation of the meridional flow; its construction is explained in Sect. 2.3. This term renders the model nonlinear.

\subsection{Flux emergence}

The source term, $S(\lambda, \phi, t)$, in Eq. (1) represents the emergence of magnetic flux at the surface in the form of bipolar magnetic regions appearing at the latitudes and longitudes of sunspot groups taken from the combined RGO/SOON sunspot record (Balmaceda et al. 2009) at the time of their maximum surface area. The sunspot group size was converted to magnetic flux flux as described in Cameron et al. (2010, and references therein), the calibration being based upon the total unsigned magnetic flux derived from the synoptic magnetic maps ${ }^{1}$ taken with the Michelson Doppler Imager (MDI) instrument onboard of the Solar Heliospheric Observatory (SoHO) spacecraft. We assumed that the latitude dependence of the initial tilt angle, $\alpha$, of the new bipolar magnetic regions has the form $\alpha=T \sqrt{\lambda}$, using $T=1.42$ for all cycles (angles measured in degrees), so that cycle-dependent changes of the tilt angles result solely from the magnetic-field dependent inflows toward the activity belts.

The transition from the RGO data covering the period up to 1976 to the subsequent SOON data involves cross-calibration issues. For instance, the minimum area required for a sunspot group to be included changed from 1 millionth of a hemisphere

1 http://soi.stanford.edu/magnetic/index6.html 
in the RGO data to 10 millionths of a hemisphere in the SOON data. In order to account for this change, Balmaceda et al. (2009) used other datasets overlapping both the RGO and SOON data to determine a correction factor of approximately 1.4 for the sunspot group areas in the SOON data (see also Wilson \& Hathaway 2005). While a global correction factor might be adequate for the total sunspot areas, applying it uniformly to individual sunspot groups of all sizes (as used in the source term of SFT simulations) is hardly justified since it probably grossly overestimates the areas of the large sunspot groups. This affects particularly strongly the variation of the polar field, to which the large sunspot groups contribute most because of their large latitude separations and fluxes. Since the overestimated sources lead to unrealistic results for the polar fields (and the closely related axial dipole component), which are at our focus of interest in this paper, we consider the SFT results only until the activity minimum between cycles 20 and 21, which nearly coincides with the termination of the RGO data in 1976.

\subsection{Latitudinal inflows}

We treated the cumulative effect of the inflows toward active regions in terms of a local perturbation, $\Delta v(\lambda, t)$, of the axisymmetric meridional flow. The amplitude of the background meridional flow was kept constant. This is consistent with the result of Cameron \& Schüssler (2010), who showed that the activeregion inflows fully explain the variations in the low-degree spherical-harmonic decomposition of the observed meridional flow (Hathaway \& Rightmire 2010), without requiring a change in the overall meridional flow amplitude. A time-independent global meridional circulation is also consistent with the finding by Hathaway (2011) that the latitudinal drift rates of the activity belts are the same for all cycles when measured with respect to the epochs of the cycle minima. The perturbation of the meridional flow in our SFT simulations was therefore modeled using almost the same procedure as in Cameron \& Schüssler (2010). Taking the instantaneous latitude profile of the azimuthally averaged unsigned radial magnetic surface field,

$\left.\langle|B|\rangle(\lambda, t)=\frac{1}{2 \pi} \int_{0}^{2 \pi} \mid B(\phi, \lambda, t)\right) \mid \mathrm{d} \phi$,

we set the inflow speed towards the activity belts proportional to the smoothed derivative of $\langle|B|\rangle$ with respect to latitude, viz.

$\Delta v(\lambda, t)=c_{0} \int\left(\frac{\cos \lambda^{\prime}}{\cos 30^{\circ}}\right) \frac{\mathrm{d}\langle|B|\rangle}{\mathrm{d} \lambda^{\prime}} \mathrm{e}^{-\left(\lambda-\lambda^{\prime}\right)^{2} / \sigma} \mathrm{d} \lambda^{\prime}$,

where the choice of $\sigma$ effects a smoothing in latitude with a full width at half maximum of $20^{\circ}$. The prefactor $c_{0}=$ $9.2 \mathrm{~m} \mathrm{~s}^{-1} \mathrm{G}^{-1} \mathrm{deg}$ was calibrated in Cameron \& Schüssler (2010) by requiring that the amplitude of the inflow should be comparable to that reported by Gizon et al. (2010) from helioseismic observations during cycle 23 . The only difference to the procedure of Cameron \& Schüssler $(2010)$ is the factor $\left(\cos \lambda / \cos 30^{\circ}\right)$ in Eq. (5), which suppresses unrealistically strong flow perturbations that would otherwise result at high latitudes from the gradient of the polar fields.

For a nonlinear SFT simulation including the effect of magnetic-field-dependent inflows, Fig. 1 shows time-latitude diagrams of the longitude-averaged radial magnetic field (signed and unsigned) as well as of the meridional flow speed and its perturbations, $\Delta v$, due to the modeled inflows. Stronger cycles are associated with bigger perturbations of the meridional flow. For very high cycle amplitude, the flow direction may even locally reverse, which can be seen most prominently during cycle 19 . Depending on the asymmetry between the magnetic field distributions in the two hemispheres, cross-equator flows occasionally arise.

\section{Results}

\subsection{Effects of the inflows on the axial dipole}

The latitudinal inflows towards the activity belts affect the nearsurface evolution of the magnetic field in a nonlinear fashion, since they depend on the magnetic field distribution and strength. To investigate the potential of this effect as a nonlinear feedback in flux-transport dynamos, we compare SFT simulations with and without inflows. A relevant quantity for the amplitude of flux-transport dynamos is the maximum axial dipole component of the surface field around activity minimum. This field represents the main part of the poloidal field from which the toroidal field of the next activity cycle is generated by differential rotation. Note that the axial dipole component is axisymmetric and therefore independent of the presence of zonal flows since any differential rotation does not affect the longitude-averaged radial surface field in a SFT simulation (e.g., Baumann et al. 2004).

The time development of the magnetic flux associated with the axial dipole, $\Phi_{\text {dipole }}$, for cycles 12 through 20 is shown in Fig. 2. Three SFT simulation runs are compared: the "reference" case (cf. Fig. 1) with calibrated inflows towards the activity belts (black line), the case without inflows (red line), and a case for which the inflows were enhanced by a factor 1.5 with respect to the reference case (blue line). The first cycle in the simulation is not considered further since it is still dominated by the initial condition (for details, see Cameron et al. 2010). In both the reference case and the case with no inflows, $\Phi_{\text {dipole }}$ peaks at or shortly before the activity minima.

For all cycles except cycles 19, we see that the inflows in the reference case tend to increase $\Phi_{\text {dipole }}$. We can understand this result by considering that the preferential cross-equator transport of leading-polarity magnetic flux in tilted bipolar magnetic regions is the essential effect that produces a flux imbalance in each hemisphere, leading to the polarity reversal and buildup of the opposite-polarity axial dipole field (e.g., Cameron \& Schüssler 2007). There are two opposing effects of the latitudinal inflows on the cross-equator transport: 1) for low-latitude active regions, the inflows are broad enough to cross the equator and thus enhance the flux transport from the other hemisphere, increasing the flux imbalance and thus eventually strengthening the axial dipole; and 2) the convergence of the inflows reduces the tilt angle, counteracting the diffusive latitudinal spreading of a bipolar region, and thus weakening its eventual contribution to the axial dipole. For weak to moderate cycles, the first effect dominates and the enhanced cross-equator transport leads to stronger axial dipole fields around activity minima. For stronger cycles, the combination of the Waldmeier effect (whereby stronger cycles peak earlier) and the fact that activity starts at higher latitudes before propagating towards the equator results in the activity belts being further away from the equator during the maximum phase of such cycles. This suppresses the flux transport by cross-equatorial flows, so that eventually the opposing effect 2) becomes dominant: the inflows act against the diffusion to reduce the tilt angle and thus reduce the diffusive cross-equatorial transport of magnetic flux. Figure 2 shows this clearly for the minimum periods between cycles 19, 20, and 21: while the maxima of the axial dipole flux in the case without inflows (red curve) largely follow the amplitudes of the 

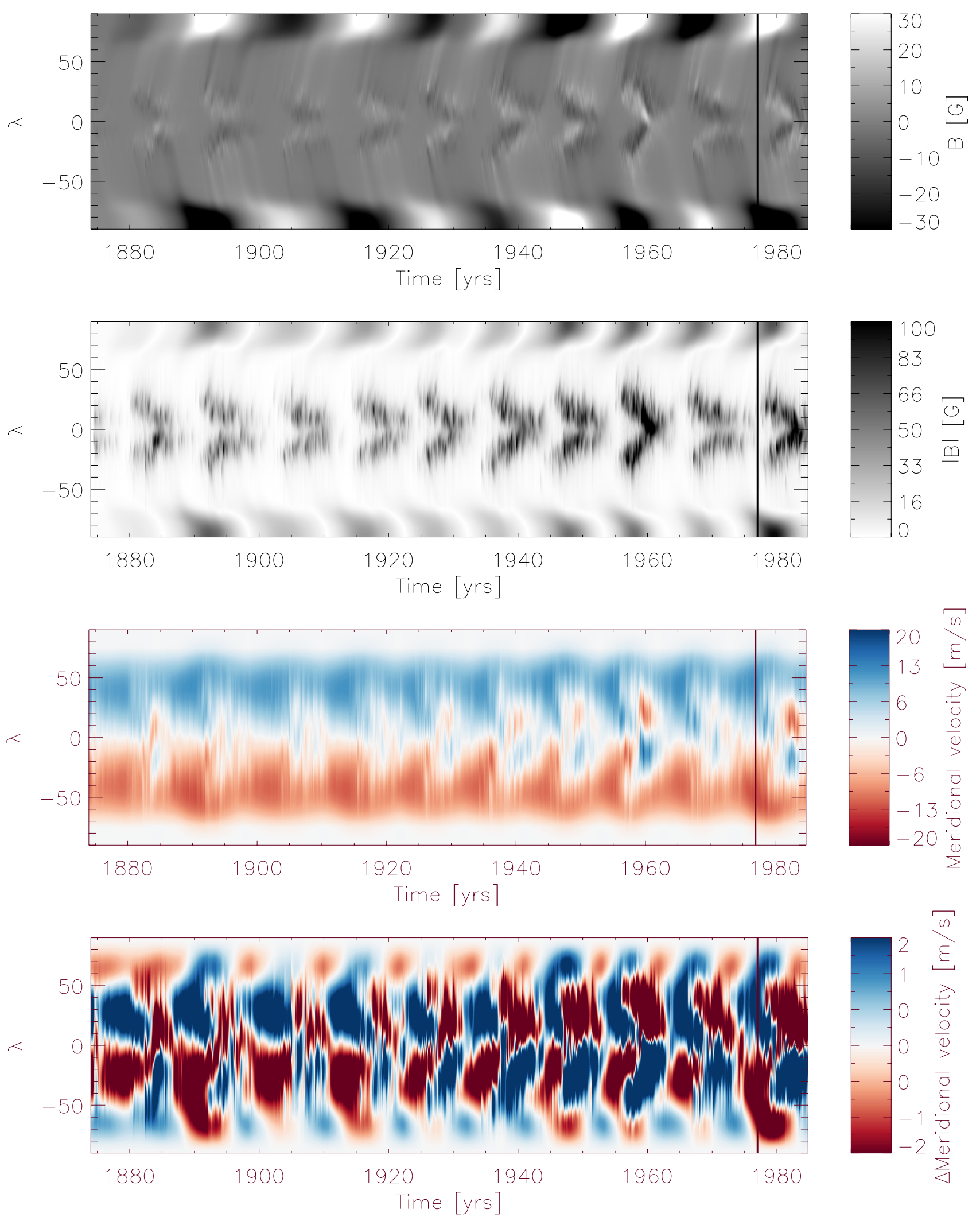

Fig. 1. Time-latitude diagrams of various quantities from a SFT simulation run with magnetic-field dependent meridional inflows towards the activity belts. From top to bottom: longitudinally averaged signed radial magnetic field, longitudinally averaged unsigned radial magnetic field, meridional flow velocity, and meridional flow perturbation $\left(\Delta v(\lambda, t)\right.$, saturated for values exceeding $\left.\pm 2 \mathrm{~m} \mathrm{~s}^{-1}\right)$. Black vertical lines indicate the termination of the RGO sunspot data in 1976. 
R. H. Cameron and M. Schüssler: Strengths of solar cycles

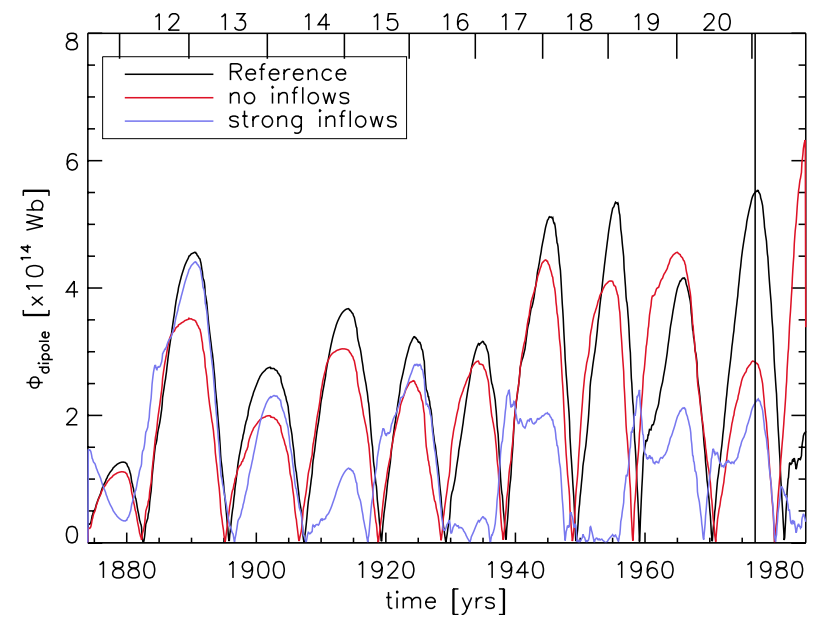

Fig. 2. Magnetic flux corresponding to the axial dipole, $\Phi_{\text {dipole }}$, from the SFT simulation with inflows (reference case, black curve), without inflows (red curve), and with strong inflows (amplitude multiplied by 1.5 with respect to the reference case, blue curve). The black vertical line indicates the termination of the RGO sunspot data in 1976.

preceding cycles in a linear manner, including the inflows (black curve) leads to a weakened axial dipole after cycle 19 and a much stronger dipole after cycle 20.

We studied the sensitivity of the axial dipole on the strength of the inflows by running SFT simulations with inflow amplitudes multiplied by factors of 0.5 and 1.5 , respectively, with respect to the reference case. While in the case of weakened inflows the time evolution of $\Phi_{\text {dipole }}$ is essentially the same as in the case of no inflows (red curve in Fig. 2), for the enhanced inflows the deviations from the reference case are drastic (blue curve in Fig. 2). The overly strong reduction of the cross-equator flux transport in this case leads to some almost failed polar field reversals and a resulting strong 22 -year periodicity of $\Phi_{\text {dipole }}$.

For a comparison of the SFT models with empirical results, Fig. 3 shows the relation between the maxima of $\Phi_{\text {dipole }}$ from our SFT simulations and the open heliospheric magnetic flux during these periods determined from the variations of the geomagnetic $a a$-index (Lockwood et al. 2009). We may safely assume that the open magnetic flux during activity minimum periods is strongly dominated by the axial dipole (e.g., Wang \& Sheeley 2009). The upper panel of Fig. 3 gives the result for the simulation without inflows, which shows a weak $(r=0.55)$ and insignificant $(p=0.15)$ correlation; there is also a clear deviation of the regression line (solid) from the bisectrix (dashed). In contrast, for the reference case with inflows (lower panel of Fig. 3) we find a good correlation $(r=0.74, p=0.022)$ and a regression line nearly coinciding with the bisectrix. It is obvious from the blue curve in Fig. 2 that the case with enhanced inflows is inconsistent with the empirically determined open fluxes.

\subsection{Relation between axial dipole and cycle strength}

There is an empirical correlation between quantities related to the polar fields around activity minimum and the amplitude of the subsequent activity cycle. For instance, considering the values of the open heliospheric flux near activity minimum as inferred from geomagnetic variations (Lockwood et al. 2009) and the maximum sunspot number (based on smoothed monthly
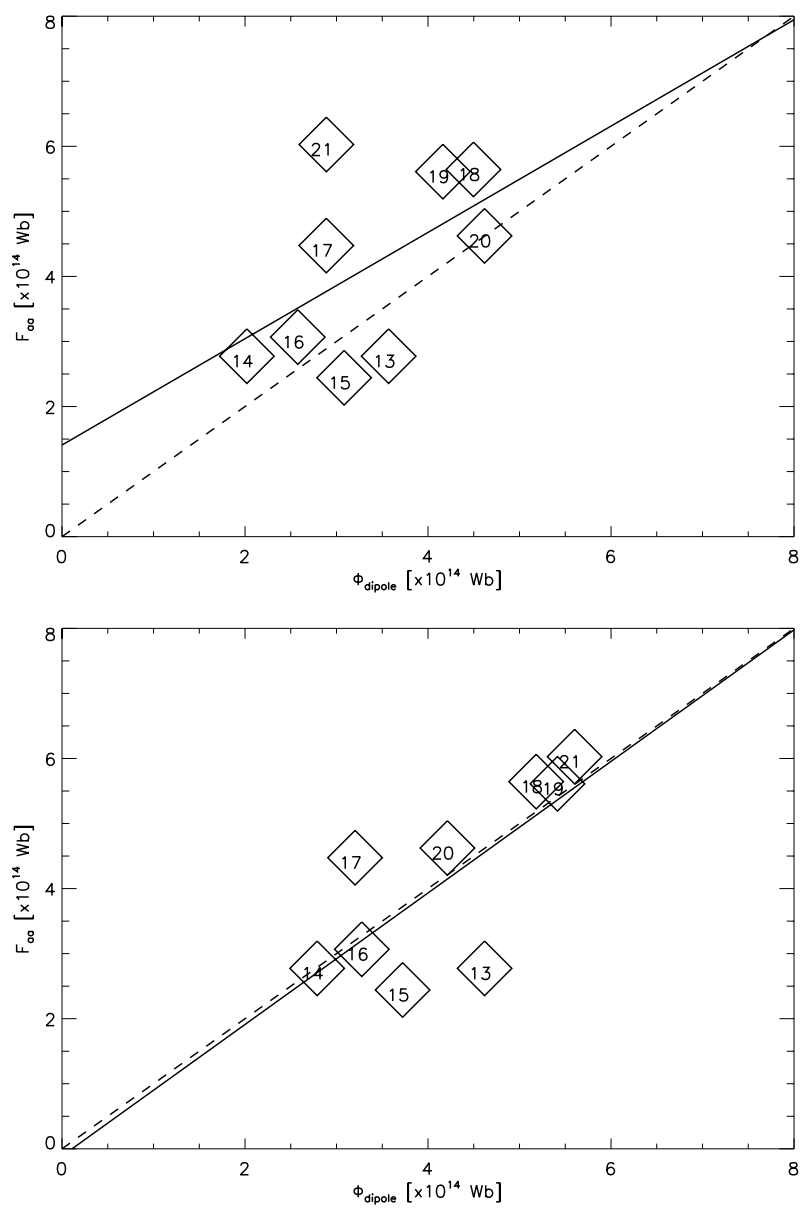

Fig. 3. Maxima of $\Phi_{\text {dipole }}$ from SFT simulations vs. open flux during activity minima as determined from the measured geomagnetic $a a$-index (Lockwood et al. 2009). Upper panel: case without inflows. Lower panel: reference case with inflows. Numbers within symbols give the index of the cycle following the respective activity minimum. Full lines indicate regression curves, dashed lines the bisectrix of the first quarter plane.

mean values ${ }^{2}$ ) of the subsequent cycle, $R_{\max }$, we find $r=0.87$ $(p=0.0025)$ for cycles $13-21$. This is consistent with the results of Wang \& Sheeley (2009). On the other hand, the correlation with the maximum sunspot number of the preceding cycle is insignificant $(r=0.55, p=0.12)$. These results indicate that the polar fields reflect the poloidal source of the toroidal field of the next cycle, which is consistent with the interpretation of the solar cycle in terms of a BL-type dynamo.

In order to evaluate the effect of the latitudinal inflows on this correlation and thus infer their viability as a nonlinear feedback mechanism for BL-type dynamos, we consider the correlations between the maxima of $\Phi_{\text {dipole }}$ as provided by SFT simulations and the values of $R_{\max }$ of the subsequent and preceding cycles, respectively. Figure 4 shows these relations for the simulations with and without inflows, respectively. In latter case, the correlation is moderate for $R_{\max }$ of the preceding cycle (upper left panel; $r=0.68, p=0.044)$ and insignificant for the subsequent cycle (upper right panel; $r=0.54, p=0.13$ ), which is in striking contrast to the empirical correlations. Including the inflows completely changes the picture: while the correlation of $\Phi_{\text {dipole }}$ with $R_{\max }$ of the preceding cycle becomes insignificant

\footnotetext{
2 ftp://ftp.ngdc.noaa.gov/STP/SOLAR_DATA/SUNSPOT_ NUMBERS/docs/maxmin. new
} 

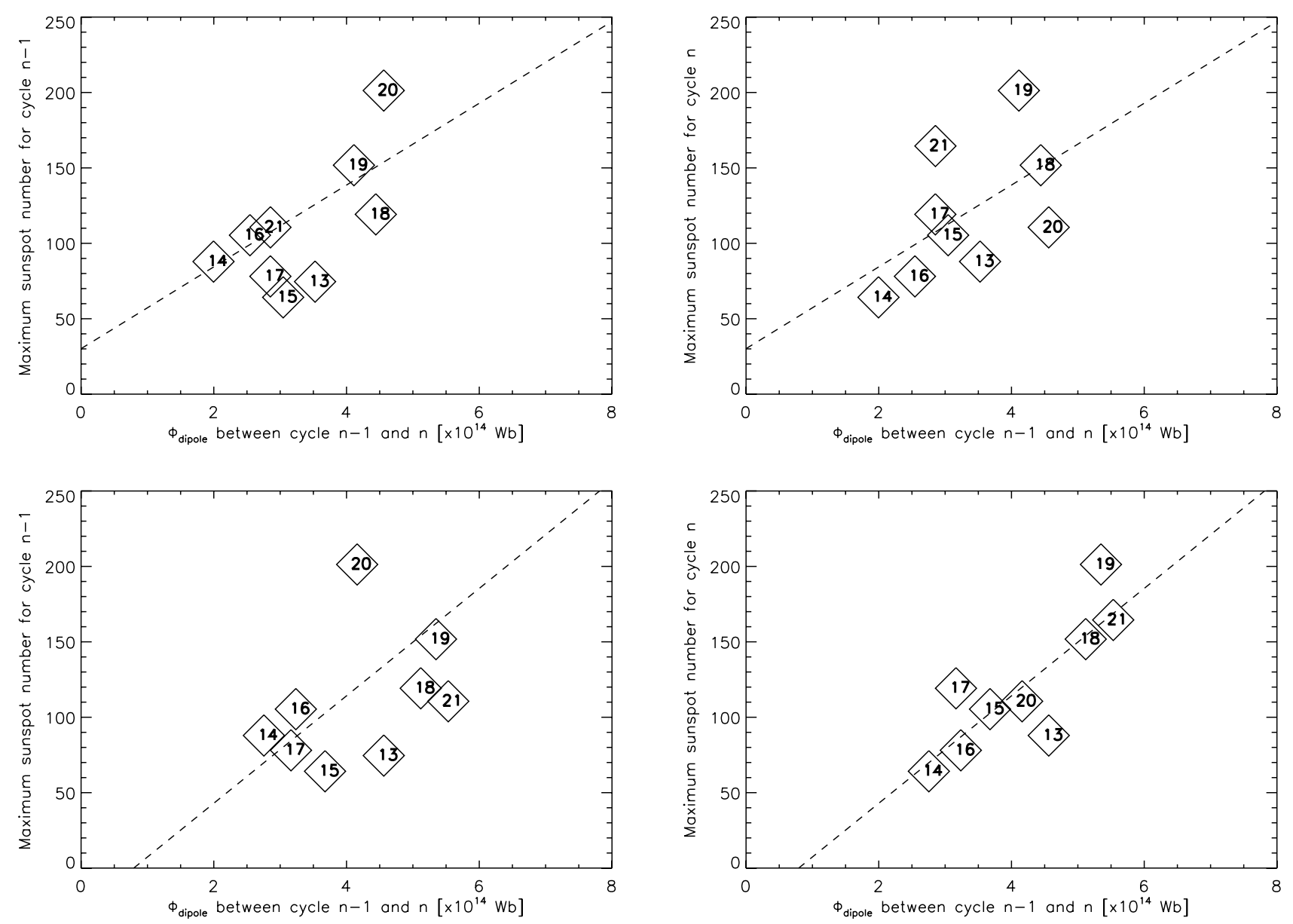

Fig. 4. Correlation diagrams for the maxima of $\Phi_{\text {dipole }}$ during activity minima between cycles $n-1$ and $n$ from the SFT simulation vs. the maxima of the sunspot number of the the cycle preceding the minimum $(n-1$, left panels) and of the subsequent cycle ( $n$, right panels), respectively. Upper row: case without inflows; lower row: reference case with inflows. Numbers within symbols give the index, $n$, of the cycle following the respective activity minimum. Linear regressions are indicated by the dashed lines.

(lower left panel; $r=0.38, p=0.31$ ), the correlation with $R_{\max }$ of the subsequent cycle is highly significant in this case (lower right panel; $r=0.82, p=0.0065$ ), which is comparable to the empirical correlation based on the open heliospheric flux.

These results show that the inflows towards the activity belts provide an important nonlinearity in the evolution of the Sun's poloidal field at the solar surface. This conclusion follows essentially from the magnitude of the modeled inflows and is largely independent of the model parameters. Furthermore, our results strongly suggest that the latitudinal inflows towards the activity belts have a dominant influence on the strength of the subsequent cycle and provide a nonlinear feedback mechanism for a BL-type dynamo. While other nonlinear processes might also play a role, the high correlations found here suggest that their effect is probably rather limited. Further work is going to assess the extent to which the good correlation with the observed cycle strengths found here depends on the SFT parameters and on our model of the inflows. Here we simply note that the parameters used in this study are essentially those of Cameron et al. (2010, SFT parameters) and of Cameron \& Schüssler (2010, inflow model), which all are based on observational constraints.

The high sensitivity of the axial dipole strength on the amplitude of the inflows is consistent with the large variation of the cycle amplitudes in the historical record, indicating also that rather weak fluctuations of the surface distribution of active regions could temporarily switch off the BL-type dynamo and may drive the system into an extended minimum state.

\section{Conclusion}

Our SFT simulations show that magnetic-field-dependent latitudinal inflows converging towards the activity belts significantly affect the build-up of the polar field by modifying the crossequator transport of magnetic flux. The resulting amplitudes of the magnetic flux contained in the axial dipole component during activity minima correlate well with the empirically derived values of the open heliospheric flux during these periods. The inflows strengthen the axial dipole in weaker cycles, for which parts of the inflows provide enhanced cross-equator transport of magnetic flux. For strong cycles, the reduction of the tilt angles of bipolar magnetic regions by the converging inflows dominates and leads to a weakening of the axial dipole. Consistent with the empirical results, the SFT simulations including latitudinal inflows show a strong correlation between the axial dipole around activity minimum and the observed maximum sunspot number of the subsequent cycle. This indicates that the inflows are a key ingredient in determining the amplitude of solar cycles by providing a nonlinear feedback mechanism for the saturation of a Babcock-Leighton-type dynamo mechanism. 
R. H. Cameron and M. Schüssler: Strengths of solar cycles

\section{References}

Balmaceda, L. A., Solanki, S. K., Krivova, N. A., \& Foster, S. 2009, J. Geophys. Res., 114, A07104

Baumann, I., Schmitt, D., Schüssler, M., \& Solanki, S. K. 2004, A\&A, 426, 1075

Baumann, I., Schmitt, D., \& Schüssler, M. 2006, A\&A, 446, 307

Caligari, P., Moreno-Insertis, F., \& Schüssler, M. 1995, ApJ, 441, 886

Caligari, P., Schüssler, M., \& Moreno-Insertis, F. 1998, ApJ, 502, 481

Cameron, R., \& Schüssler, M. 2007, ApJ, 659, 801

Cameron, R. H., \& Schüssler, M. 2010, ApJ, 720, 1030

Cameron, R. H., Jiang, J., Schmitt, D., \& Schüssler, M. 2010, ApJ, 719, 264

Charbonneau, P. 2010, Liv. Rev. Sol. Phys., 7, 3,

http: //www . livingreviews .org/lrsp-2010-3

Choudhuri, A. R. 2008, J. Astrophys. Astron., 29, 41

Dasi-Espuig, M., Solanki, S. K., Krivova, N. A., Cameron, R., \& Peñuela, T. 2010, A\&A, 518, A7

D'Silva, S., \& Choudhuri, A. R. 1993, A\&A, 272, 621

Fan, Y. 2009, Liv. Rev. Sol. Phys., 6, 4,

http://www . livingreviews . org/lrsp-2009-4

Fan, Y., Fisher, G. H., \& McClymont, A. N. 1994, ApJ, 436, 907

Gizon, L. 2004, Sol. Phys., 224, 217

Gizon, L., \& Birch, A. C. 2005, Liv. Rev. Sol. Phys., 2, 6

Gizon, L., \& Rempel, M. 2008, Sol. Phys., 251, 241

Gizon, L., Birch, A. C., \& Spruit, H. C. 2010, ARA\&A, 48, 289
González Hernández, I., Howe, R., Komm, R., \& Hill, F. 2010, ApJ, 713, L16

Haber, D. A., Hindman, B. W., Toomre, J., et al. 2002, ApJ, 570, 855

Hathaway, D. H. 2011, Sol. Phys., 273, 221

Hathaway, D. H., \& Rightmire, L. 2010, Science, 327, 1350

Howard, R., \& Labonte, B. J. 1980, ApJ, 239, L33

Jiang, J., Işik, E., Cameron, R. H., Schmitt, D., \& Schüssler, M. 2010, ApJ, 717, 597

Jiang, J., Cameron, R. H., Schmitt, D., \& Schüssler, M. 2011a, A\&A, 528, A82 Jiang, J., Cameron, R. H., Schmitt, D., \& Schüssler, M. 2011b, A\&A, 528, A83

Layden, A. C., Fox, P. A., Howard, J. M., Sarajedini, A., \& Schatten, K. H. 1991, Sol. Phys., 132, 1

Lockwood, M., Rouillard, A. P., \& Finch, I. D. 2009, ApJ, 700, 937

Schatten, K. H., Scherrer, P. H., Svalgaard, L., \& Wilcox, J. M. 1978, Geophys. Res. Lett., 5, 411

Schüssler, M. 2007, Astron. Nachr., 328, 1087

Snodgrass, H. B. 1983, ApJ, 270, 288

Spruit, H. C. 2003, Sol. Phys., 213, 1

Švanda, M., Kosovichev, A. G., \& Zhao, J. 2008, ApJ, 680, L161

van Ballegooijen, A. A., Cartledge, N. P., \& Priest, E. R. 1998, ApJ, 501, 866

Wang, Y.-M., \& Sheeley, N. R. 2009, ApJ, 694, L11

Wilson, R. M., \& Hathaway, D. H. 2005, NASA STI/Recon Technical Report N, 62,22159

Zhao, J., \& Kosovichev, A. G. 2004, ApJ, 603, 776 